ing a sequence of moves, each of which is random but with probabilities that should optimize expected gains. These elegant theoretical results have long been known to provide poor fits to laboratory data from real players.

The success of their model is impressive, but, as they point out, it is only part of the significance of their results. The three best-performing models are (i) a model drawn from the previous literature known as Normalized Fictitious Play (7), (ii) the simplest MarchioriWarglien model, and (iii) a variant of their model with one free parameter. Although they vary in how it is incorporated, all three are regret-driven learning models.

To these results we can add others $(8)$ showing that a regret-based model can also account for puzzling empirical patterns in various forms of auction bidding. As Marchiori and Warglien recognize, the superior fit of these regret-based models aligns very nicely with results from recent neuropsychological work, where studies of brain activity during decision-making have pointed increasingly to the crucial role played by brain areas implicated in feelings of regret (9). In particular, regret involves constructing a counterfactual image of what the actor would have felt if he or she had acted differently. Psychological subjects with impaired abilities to construct such felt counterfactuals - for example, because of damage to brain areas such as the orbitofrontal cortex - are observed to behave quite differently from normal subjects in choice situations (10).

Although the Marchiori-Warglien model gives a central role to regret-driven learning in making its successful predictions, it is important to recognize that the learning in their model is an approximation of the learning dynamics of experimental players, just as the regret is an approximation of the complex feelings of success and failure experienced by the players. The adaptive neural net structure used in their model converges toward its stable propensities for long-run play fairly quickly, generally in fewer iterations than experimental subjects require. Thus, the data on play that it fits least well are the cases where subjects take the longest to stabilize their patterns of play. Other models, typically those based on more traditional reinforcement learning, have been found to do better at tracking the early stages of experimental play (5). But versions of those reinforcement models that were included in the present work did less well overall than any of the regret-driven models.

The great virtue of the parsimony and rigor of economic theorizing is exactly that improvements often accumulate rapidly. Thus, the Marchiori-Warglien model may not be the final word in this development of psychologically plausible approximations for economic game play (11). However, the authors have made an important contribution with their psychologically grounded insight that regret-driven learning provides the best approximating form to date for some of the most recalcitrant economic laboratory data that we have. And their use of a neural network structure incorporating payoffs has interesting possibilities for generalization to other classes of games. They have made a step that both takes us forward and simultaneously helps to define the road that lies ahead.

References and Notes

1. D. Marchiori, M. Warglien, Science 319, 1111 (2008).

2. C. Camerer, U. Malmendier, in Economic Institutions and Behavioral Economics: Proceedings of the Yrjö Jahnsson Foundation 50th Anniversary Conference, P. Diamond, H. Vartiainen, Eds. (Princeton Univ. Press, Princeton, N], 2007), pp. 235-280.

3. M. D. Cohen, in Economic Institutions and Behavioral Economics: Proceedings of the Yrjö Jahnsson Foundation 50th Anniversary Conference, P. Diamond, H. Vartiainen, Eds. (Princeton Univ. Press, Princeton, N], 2007), pp. 281-290.

4. E. Fehr, C. Camerer, Trends Cogn. Sci. 11, 419 (2007).

5. I. Erev, A. E. Roth, R. L. Slonim, G. Barron, Econ. Theory 33, 29 (2007).

6. R. Gibbons, Game Theory for Applied Economists, (Princeton Univ. Press, Princeton, N], 1992).

7. I. Erev, A. E. Roth, Am. Econ. Rev. 88, 848 (1998).

8. R. Engelbrecht-Wiggans, E. Katok, Econ. Theory 33, 81 (2007).

9. G. Coricelli, R. J. Dolan, A. Sirigu, Trends Cogn. Sci. 11, 258 (2007).

10. A. Bechara, H. Damasio, A. Damasio, Cereb. Cortex 10, 295 (2000).

11. For example, the few cases that the simple model has trouble predicting are ones in which the optimal behavior is fairly close to an unmixed, or pure strategy, suggesting that there are further regularities that may be captured in a subsequent variation on the model.

10.1126/science. 1155477

\title{
Bioinspired Structural Materials
}

\section{Christine Ortiz and Mary C. Boyce}

$\mathrm{T}$ he huge diversity of structural biological materials that exist in nature, even within a single species, and the complexity, multifunctionality, and multiscale nature of their structure-property relationships has been studied extensively for decades (1). Using materials available in the environment that typically exhibit poor macro-scale mechanical properties (brittle biological ceramics and compliant macromolecules), they can achieve orders-of-magnitude increases in strength and toughness; in many cases, this "mechanical property amplification" occurs in a nonadditive manner that goes

Department of Materials Science and Engineering, Massachusetts Institute of Technology, Cambridge, MA 02139, USA. E-mail: cortiz@mit.edu beyond the simple composite rule of mixture formulations. Synthetic structural materials that take advantage of the mechanical design principles found in nature could transform many fields; e.g., materials science, mechanical and civil engineering, and aeronautics and astronautics. Here, we highlight a few recent developments in this area and summarize unexplored opportunities for the future.

Bonderer et al. [page 1069 of this issue (2)] have carried out the deliberate microstructural design of a multilayered alumina platelet-reinforced chitosan nanocomposite, inspired by the inner nacreous layer of many seashells. The alumina platelets possess higher ultimate tensile strength than the aragonite platelets found in nacre; this reflects a general design concept, whereby the weak constituents found
Materials scientists are seeking to create synthetic materials based on the mechanical design principles found in biological materials such as seashell nacre.

in nature are replaced with more advanced synthetic engineered materials, with the goal of producing structural composite materials with mechanical properties that exceed both those of nacre and those of state-of-the-art synthetic materials.

The thickness of the alumina platelets was chosen to be similar to that found in nacre $(\sim 200$ nanometers). At such sub-micrometer thicknesses, the strength of brittle materials often increases compared with the bulk material because of the decreasing size and probability of flaws (3). The average platelet aspect ratio was selected based on a classic composite shear lag load transfer model (4); this critical length maximizes the load transferred to the platelet while averting platelet fracture and instead favors a failure mechanism of platelet pullout 
and matrix shear (as observed in nacre). The authors also achieved excellent alignment and dispersion of the platelets in the matrix up to volume fractions of 0.2 , using a colloidal-based technique. Tensile mechanical properties were amplified in the composite material compared to the chitosan matrix, and significant plasticity was retained, suggesting pathways for extensive deformation of the matrix at these volume fractions before platelet pullout. The authors have thus created a material that is simultaneously stiff, strong, and tough by via constituents of appropriate length scale and geometry, using mechanical design principles derived from nature, as well as achieving dispersion of the reinforcing component in the matrix.

Podsiadlo and colleagues (5) have taken an alternative approach in the design of a montmorillonite clay platelet-poly(vinyl alcohol) matrix nacre-mimetic artificial nanocomposite by focusing on tailoring the chemistry of the platelet-matrix interface to enhance load transfer. Biological composites make use of
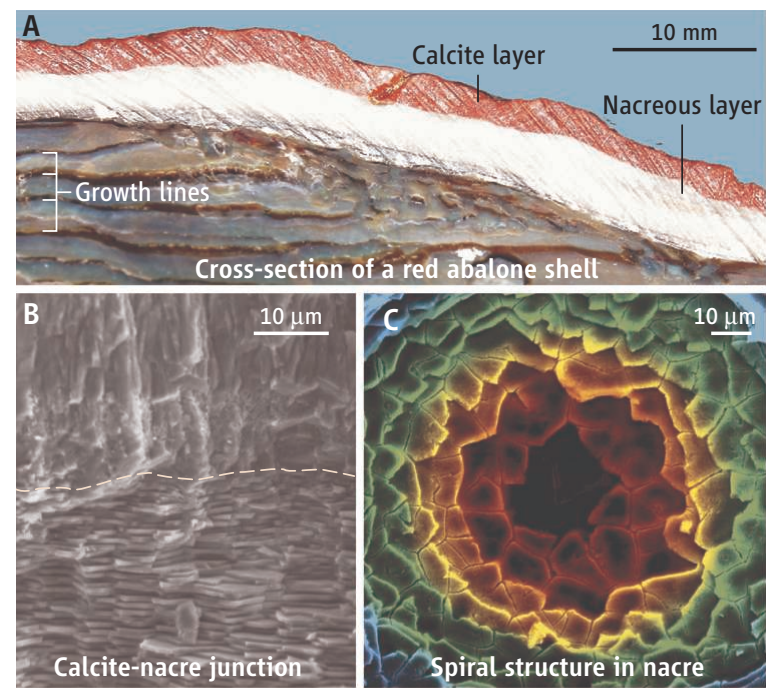

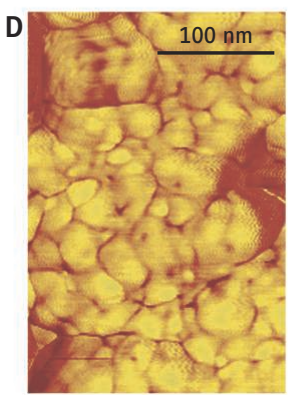

Surface of a nacre platelet

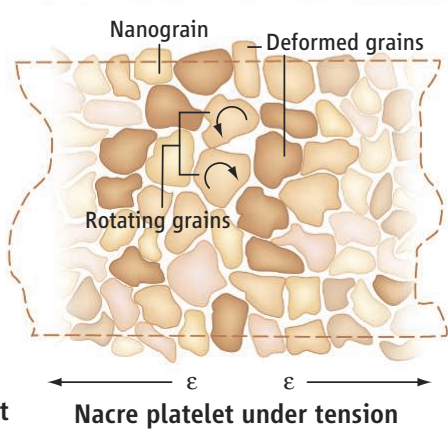

Nacre platelet under tension
Multiscale mechanical design principles of seashells. (A) Multilayered structure of the cross-section of California red abalone shell (10). (B) Artificially colorized scanning electron microscopy (SEM) image of the calcite-nacre junction region in a Trochus niloticus shell (9). (C) Artificially colorized SEM image of a layer-to-layer spiral in T. niloticus nacre. (D) (Left) Atomic force microscopy image of surface nanograins on an individual nacre platelet from California red abalone and (right) schematic of nanograin rotation under tension (13). local chemistry, compositional gradients, macromolecular supramolecular structure, length scale effects, geometry, and other factors to design robust interfaces and interphases that bond together different material phases, even in the presence of water ( 6 ). In cross-linking Gluteraldehyde treatment created additional covalent acetal bridges between the matrix and platelets, and dramatic increases in stiffness and strength were hieved compared to the matrix material.

Another example of nacre-mimetic interfacial design between structural elements in a nanocomposite has been reported by Tang et al. (7). anically bonded polyelectrolyte matrix " sa mimic the high toughness extenprofile due to sacrificial noncovalent bonding observed experimentally in the organic component of nacre (8) and modeled theoretically by Qi et al. (9). This unique interfacial property governs the adhesive matrix behavior, thus controlling and mitigating shear lag load transfer to the aragonite tablets, averting failure of both the tablets and the organic adhesive layers, and increasing ductility and energy dissipation.

Even in the limited context of nacre, many design principles remain to be explored. The ability to access a wide range of volume fractions of the reinforcing component will enable additional design optimization in terms of tailoring stiffness, strength, and toughness. Recent theoretical models (10) and electron microscopy studies (11) of nacre have shown that additional microstructural features - platelet layer offset, platelet surface waviness, the Voronoi arrangement of platelets in each plane, and screw dislocations leading to large, interconnected layer-tolayer spiral structures (see the figure, panel C) and a tessellated zigzag morphology - may all play a role in the biomechanical functionality.

The smaller and larger length scale design principles of seashells have received less attention. At the smaller length scales, individual nacre platelets are complex organic-inorganic com- posites with a unique sector structure; each sector possesses nanometer-sized surface domains or asperities $(12,13)$. Individual platelets can exhibit considerable plasticity before fracture upon penetration by an indenter (12), and the constituent surface "nanograins" can deform and rotate under an applied tensile load (13) (see the figure, panel D). In addition, there is a complex three-dimensional distribution of organic matrix components spatially within the plane parallel to the nacre tablets (14).

At the larger length scale of the shell (see the figure, panel A), the multilayered structure, the prismatic calcite outer layer, integrity of the calcite-nacre interface (see the figure, panel B), confinement effects between the layers, structure and property gradation within and between layers, and anisotropy of the layers all work collectively to provide enhanced mechanical performance.

Last, we have yet to fully understand and take advantage of the inherent specificity of natural mechanical design principles. For example, multilayered armored fish scales serve as protection from predatory penetrating impacts (15), mussel byssal threads are hysteretic yet resilient to large strain deformation in order to maintain adhesion to rocks in the face of the pounding surf (16), and graded layer junctions in teeth resist catastrophic fracture during mastication (17). Each of these systems experiences, and has been designed to endure, very different loading conditions in their environment and during their function.

References and Notes

1. S. A. Wainwright, W. D. Biggs, ]. D. Currey, ]. M. Gosline, Mechanical Design in Organisms (Princeton Univ. Press, Princeton, N], 1976).

2. L. ]. Bonderer, A. R. Studart, L. ]. Gauckler, Science 319, 1069 (2008).

3. H. Gao, B. Ji, I. L. Jager, E. Arzt, P. Fratzl, Proc. Natl. Acad. Sci. U.S.A. 100, 5597 (2003).

4. A. P. Jackson, J. F. V. Vincent, R. M. Turner, Proc. R. Soc. London Ser. B. 234, 415 (1998).

5. P. Podsiadlo et al., Science 318,80 (2007).

6. S. Weiner, F. Nudelman, E. Sone, P. Zaslansky, L. Addadi, Biointerphases 1, 12 (2006)

7. Z. Y. Tang, N. A. Kotov, S. Magonov, B. Ozturk, Nat. Mat 2, 413 (2003)

8. B. L. Smith et al., Nature 399, 761 (1999).

9. H. J. Qi, B. J. F. Bruet, J. S. Palmer, C. Ortiz, M. C. Boyce, in Mechanics of Biological Tissues, G. A. Holzapfel, R. W. Ogden, Eds. (Springer, Graz, Austria, 2005), pp. 175-190.

10. F. Barthelat, H. Tang, P. D. Zavattieri, C. M. Li, H. D. Espinosa, J. Mech. Phys. Solids 55, 306 (2007)

11. N. E. Yao, A. Akey, J. Mat. Res. 21, 1939 (2006).

12. B. J. F. Bruet et al., J. Mat. Res. 20, 2400 (2005)

13. X. Li, Z. H. Xu, R. Wang, NanoLetters 6, 2301 (2006).

14. F. Nudelman, B. A. Gotliv, L. Addadi, S. Weiner, J. Struc. Biol. 153, 176 (2006).

15. J. Daget, M. Gayet, F. J. Meunier, ].-Y. Sire, Fish Fisheries 2, 113 (2001)

16. E. Carrington, ]. M. Gosline, Am. Malacological Bull. 18, 135 (2004).

17. V. Imbeni, ]. J. Kruzic, G. W. Marshall, R. O. Ritchie, Nat. Mat. 4, 229 (2005).

18. The authors would like to acknowledge funding from the U.S. Army (DAAD-19-02-D0002).

10.1126/science. 1154295 